\title{
Bisphenol A disrupts inflammatory responses via Nod-like receptor protein 3 pathway in macrophages
}

\author{
Hee-Weon Lee ${ }^{1,2^{*}} \mathbb{0}$, Sang Keun $\mathrm{Ha}^{1,3}$ and Yoonsook Kim ${ }^{1}$
}

\begin{abstract}
Bisphenol A (BPA) is a harmful endocrine disruptor that is found in polycarbonate plastics such as plastic food containers and in epoxy resins such as dental resins. In the current study, we investigated the effect of BPA on function of inflammatory responses involving activation of Nod-like receptor protein 3 (NLRP3) inflammasome. Treatment with BPA decreased nitric oxide (NO) production and expression levels of inducible $\mathrm{NO}$ synthase (iNOS), prostaglandin $\mathrm{E}_{2}$ $\left(\mathrm{PGE}_{2)}\right.$, and cyclooxygenase (Cox)-2 in RAW 264.7 macrophages. BPA also suppressed activation of mitogen-activated protein kinases (MAPKs) and nuclear factor-kappa B activity (NF-KB). BPA significantly down-regulated the secretion of pro-inflammatory cytokines including tumor necrosis factor (TNF)-a, interleukin (IL)-6, IL-1 $\beta$, and IL-18. The decreased production of IL-1 $\beta$ and IL-18 induced by BPA was associated with inactivation of the activity of the NLRP3 inflammasome. Collectively, these data suggested that BPA could act as a disruptor of the inflammation activity by regulating the NF-KB/MAPK pathways and NLRP3 inflammasome activation.
\end{abstract}

Keywords: Bisphenol A, Inflammation, Macrophage, NLRP3 inflammasome

\section{Introduction}

In recent years, it has been shown that many environmental chemicals can disrupt the action of hormones. These chemicals are referred to as endocrine-disrupting chemicals (EDCs) or endocrine disruptors [1] and they mimic the role of endocrine hormones and interfere with their function. Therefore, the endocrine function of the body is changed by such an endocrine like disruptor. Although many studies have focused on metabolic abnormalities such as obesity through blocking of hormonal action and disruption of biological activity by endocrine disruptors, there are few studies on the mechanism of inflammation, which is an important factor causing various metabolic diseases. In addition, numerous studies have found that EDCs can affect immune

\footnotetext{
*Correspondence: 07919@kfri.re.kr

${ }^{1}$ Korea Food Research Institute, 245, Nongsaengmyeong-ro, Iseo-myeon,

Wanju-gun, Jeollabuk-do 55365, Republic of Korea

Full list of author information is available at the end of the article
}

cells in hormone-related diseases including rheumatoid arthritis, atherosclerosis, and diabetes [2, 3].

Bisphenol A (BPA) is representative endocrine hormone-like disruptor, which is widely used in the production of polycarbonate plastic, dental materials, medical devices and containers.

Inflammation is an innate immune response to harmful substances, immune cells, and tissue injury. It acts as an important regulator in the signaling pathways of many diseases and disorders pathogenesis, including metabolic disorders, cancer, and bacterial infections. Inflammatory responses are mediated by several different immune cells which are known to play essential roles in host defense, inflammatory responses, and immune responses. For example, macrophages are immune cells, which have a crucial role in defending against invading factors and maintaining homeostasis in the body $[4,5]$.

Macrophages have a pivotal role in regulating inflammatory disorders by producing pro-inflammatory cytokines including pro-inflammatory cytokines through immune responses with $\mathrm{T}$ cells and $\mathrm{B}$ cells. In addition, 
macrophages maintain the balance in the body by regulating inflammatory mediators including ROS production regulation in immune dysfunction. Macrophages can elicit either pro-inflammatory or anti-inflammatory responses and in this way can contribute to either tissue damage or regeneration. Modulation of the macrophage inflammatory response may be one way to treat inflammatory diseases [6].

Inflammasomes are important factors that mediate inflammation in the immune response of macrophages. Therefore, many studies have been researched on the formation and regulation of inflammation caused by inflammasomes in various diseases. NLRP3 inflammasome is molecular complexes composed of NLRP3 sensor protein, ASC adapter protein, and pro-caspase 1 protein units. Among the inflammasome components, the characteristics and roles of the inflammasome depending on the type of sensor protein [7-9].

NLRP3 inflammasomes are essential components of the mechanisms of immune signaling that regulates the maturation of pro-caspase 1 and the release of proinflammatory cytokines IL-1 $\beta$ and IL-18 in response to pathogen and molecular damages. Nevertheless, the abnormal activity of the NLRP3 inflammasome is involved in various inflammatory diseases, including cryopyrin-related periodic syndrome, cancer, metabolic diseases and rheumatoid arthritis. NLRP3 inflammasomes are stimulated by various stimuli such as a variety of intracellular events, such as mitochondrial dysfunction, free radical production, and lysosomal damage. However, it is not fully understood how the inflammatory response of NLRP3 is caused by an endocrine disruptor [10,11].

In the present study, we investigated the effect of BPA on inflammation mechanisms of macrophages. Specifically, we evaluated whether BPA affected the MAPK/ NF- $\mathrm{kB}$ signaling pathways and the NLRP3 inflammasome activity.

\section{Materials and methods Chemical reagents and antibodies}

Bisphenol A was purchased from Sigma-Aldrich (St. Louis, MO, USA). Dulbecco's modified Eagle's medium (DMEM), and fetal bovine serum (FBS) were obtained from Gibco (BRL, Carlsbad, USA). Almost chemicals, including MAPK inhibitors, and NF- $\mathrm{kB}$ inhibitor, were obtained from Sigma Chemical Co. unless otherwise stated. BPA was dissolved in distilled water and diluted at the indicated concentrations. Antibodies against target molecules were purchased from Cell signaling (Danvers, MA, USA): iNOS (D6B6S), TNF- $\alpha$ (D2D4), IL-6 (D5W4V), IL-1 $\beta$ (D6D6T), IL-18 (E8P5O), NLRP3 (D4D8T), ASC (D2W8U), Caspase-1 (ASP296), p-p65 (93H1), IkB $\alpha$ (44D4), $\beta$-actin (8H10D10) and Santa Cruz
Biotechnology (Santa Cruz, CA, USA): COX-2 (29), p65 (F-6).

\section{Cell culture}

The murine macrophage cell line was purchased from ATCC (Manassas, VA, USA). Macrophages were sustained in DMEM supplemented with $10 \%$ heat-inactivated FBS. RAW 264.7 macrophages were incubated at $37{ }^{\circ} \mathrm{C}$ in a humidified incubator containing $5 \% \mathrm{CO}_{2}$, and the cells were sub-cultured once every two days. Macrophages were stimulated with BPA for several lengths of time (4-24 h). After treatment, the macrophages were lysed with radioimmunoprecipitation assay buffer or homogenization buffer.

\section{Cell viability}

This is a test method using the principle that mitochondria reduce MTT to insoluble formazan. Cell viability was evaluated over a period of $2 \mathrm{~h}$ using the MTT quantitative colorimetric assay to detect the mitochondrial activity in living cells. By dehydrogenase action, the soluble substrate MTT tetrazolium penetrates into the living cell and becomes purple in the mitochondria. The formazan crystal is dissolved in an organic solvent such as dimethyl sulfoxide (DMSO) and the absorbance is investigated by a microplate reader (Molecular Devices, Carlsbad, CA, USA). At this time, the absorbance of the formazan dye is around $540 \mathrm{~nm}$.

\section{Determination of NO production}

NO was measured by the reduction in the stable nitrite form was measured using griess reagent System. Macrophages were incubated with indicated concentrations $(0.1-10 \mu \mathrm{g} / \mathrm{mL})$ of BPA for $24 \mathrm{~h}$ in a $5 \% \mathrm{CO}_{2}$ incubator. After stimulation of BPA for 1 day, supernatant was collected and reacted with Griess reagent at room temperature, and then measured with an absorbance of $540 \mathrm{~nm}$. The measured value was expressed as $\%$ in terms of NO production rate. The absorbance at $540 \mathrm{~nm}$ was determined using a ELISA reader. The concentration of $\mathrm{NO}_{2}{ }^{-}$was calculated from a $\mathrm{NaNO}_{2}$ standard curve. The levels of $\mathrm{NO}_{2}{ }^{-}$were indicative of the amount of $\mathrm{NO}$ production.

\section{Western blotting}

Macrophages were incubated with indicated doses of BPA $(0.1-10 \mu \mathrm{g} / \mathrm{mL})$. After stimulation, the activated cells were rinsed in PBS and lysed in a homogenizer lysis buffer. The lysate was pelleted after centrifugation for $15 \mathrm{~min}$ at $15,000 \times g$ at $4{ }^{\circ} \mathrm{C}$. The protein concentration was determined using a DC protein assay kit (Bio-Rad). Quantitative proteins were analyzed by $6-15 \%$ polyacrylamide gel and transferred to the $\mathrm{NC}$ membrane. The 
Table 1 Primer sequences and real-time PCR conditions

\begin{tabular}{lll}
\hline Gene & Forward primer $\left(\mathbf{5}^{\prime} \rightarrow \mathbf{3}^{\prime}\right)$ & Reverse primer $\left(\mathbf{5}^{\prime} \boldsymbol{\rightarrow} \mathbf{3}^{\prime}\right)$ \\
\hline TNF-a & CCC TCA CAC TCA GAT CAT CTT CT & GCT ACG ACG TGG GCT ACA G \\
IL-6 & CCA CGG CCT TCC CTA CTTC & TTG GGA GTG GTA TCC TCT GTG A \\
IL-1 $\beta$ & TTG ACG GAC CCC AAA AGA TG & TGG ACA GCC CAG GTC AAA G \\
IL-18 & CTG AAG AAA ATG GAG ACC TGG AA GTA TTA CTG CGG TTG TAC AGT \\
GAPDH & TGC ATC CTG CAC CAC CAA & TCC ACG ATG CCA AAG TTG TC \\
\hline
\end{tabular}

Table 2 Sequence of siRNA used for knockdown of the NLRP3 gene

\begin{tabular}{ll}
\hline siRNA & $\begin{array}{l}\text { Sequence }\left(5^{\prime} \rightarrow 3^{\prime}\right) \\
\text { siNLRP3 }\end{array}$ \\
\hline Sense & GGUGAAAUGUACUUAAAUCUU \\
Antisense & GAUUUAAGUACAUUUCACCUU \\
\hline
\end{tabular}

protein-transferred membrane was blocked in $0.05 \% /$ Tris-buffered saline containing $5 \%$ skim milk powder for $50 \mathrm{~min}$, and reacted with the primary and secondary antibodies. These blots were developed using an enhanced chemiluminescence kit. The expression amount of each proteins was quantified using an image J program. The measured value was expressed as the average value of 3 repeated experiments and was performed in a concentration range not causing toxicity.

\section{Quantitative real time-PCR}

Macrophages were stimulated in the presence or absence with BPA $(0.1-10 \mu \mathrm{g} / \mathrm{mL})$ for $24 \mathrm{~h}$. After BPA stimulation, total RNA was isolated from cultured cells using RNA extraction kit (Kusatsu, St. Shiga, Japan) and used for cDNA synthesis. After cDNA synthesis, $10 \mu \mathrm{L}$ of SYBR green premix (BioRad), $8 \mu \mathrm{L}$ of sterile water, and $1 \mu \mathrm{L}$ each of forward and reverse primer was mixed to adjust the total volume to $20 \mu \mathrm{L}$. Fluorescence was measured at each cycle. The RT-PCR primer sequences to find the expression of cytokines were indicated in Table 1.

\section{RNA interference}

Macrophages were cultured using suitable media into 6-well plates, and the macrophages were gently treated with buffer mixed with NLRP3 siRNA (Genolution) and siRNA transfection reagent (iNtRON) for transfection of NLRP3 gene. In order to efficiently knock down the NLRP3 gene in cells, the cells were checked with various incubation times and concentrations. The most efficient conditions were confirmed, and NLRP3 siRNA was knocked down at $50 \mathrm{nM}$ for $20 \mathrm{~h}$. (Table 2).

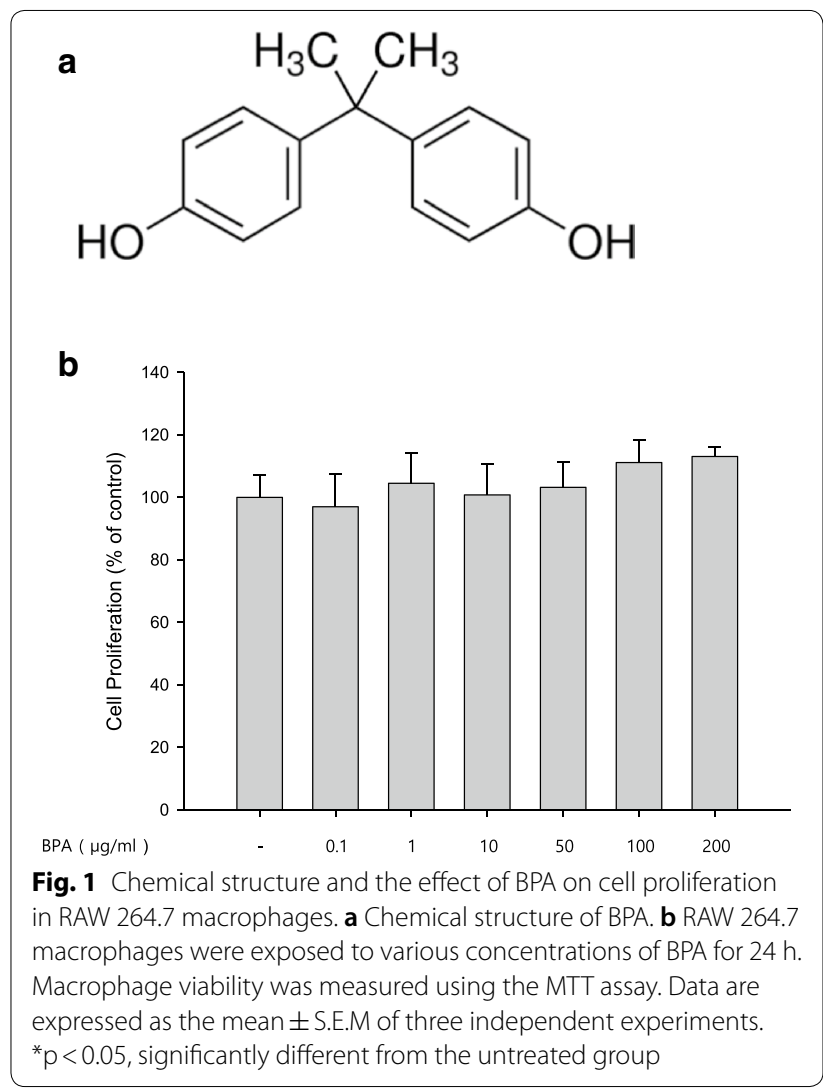

\section{Statistical analyses}

For each experiment, results were performed in triplicate and are reported as means \pm SEM. Comparisons of means between BPA-treated cells and untreated control cells were made using Student's t-test and ANOVA using the SigmaPlot 10.0 software. Significant values are indicated by an asterisk ("p $<0.05)$.

\section{Results}

Effect of BPA on RAW 264.7 macrophage proliferation

We first assessed if Bisphenol A (BPA) could affect cell proliferation under our experimental conditions (Fig. 1a). Macrophages were stimulated with the various 
concentrations $(1-200 \mu \mathrm{g} / \mathrm{mL})$ of BPA for $24 \mathrm{~h}$. Cell viability was then examined using an MTT assay. BPA at concentrations from 1 to $200 \mu \mathrm{g} / \mathrm{mL}$ did not significantly affect macrophage proliferation (Fig. 1b). Thus, we used concentrations of less than $200 \mu \mathrm{g} / \mathrm{mL}$ in all subsequent experiments.

\section{Effect of BPA on NO and PGE 2 production in RAW 264.7 macrophages}

To evaluate the effect of BPA on nitric oxide (NO) production in BPA treated macrophages, we determined nitrite levels using Griess reagents. Macrophages were activated with $0.1-10 \mu \mathrm{g} / \mathrm{mL}$ BPA for $24 \mathrm{~h}$. As shown in Fig. 2a, treatment with BPA decreased NO production in a concentration-dependent manner. In keeping with this finding, BPA also significantly suppressed the expression of inducible NO synthase (iNOS) (Fig. 2b). We also assessed the effect of $\mathrm{BPA}$ on prostaglandin $\mathrm{E}_{2}\left(\mathrm{PGE}_{2}\right)$ production and cyclooxygenase-2 (COX-2) levels in BPA treated macrophages. The level of $\mathrm{PGE}_{2}$ and the expression level of COX-2 were markedly diminished by BPA in a concentration-dependent manner (Fig. 2c, d). Collectively, our finding indicate that BPA can affect the function of macrophages by down-regulating the production of inflammatory mediators.

\section{Effect of BPA on cytokine production in RAW 264.7 macrophages}

We next examined the effects of BPA on the generation of several cytokines in RAW 264.7 macrophages. Macrophages were incubated with BPA at $0.1-10 \mu \mathrm{g} / \mathrm{mL}$ for $24 \mathrm{~h}$. BPA significantly decreased the release of inflammatory cytokines such as TNF- $\alpha$, IL- 6 , IL- $1 \beta$, and IL-18 in a concentration-dependent manner (Fig. 3a). Moreover, we evaluated whether BPA could affect cytokine expression at the transcriptional level using qRT-PCR analysis. As a

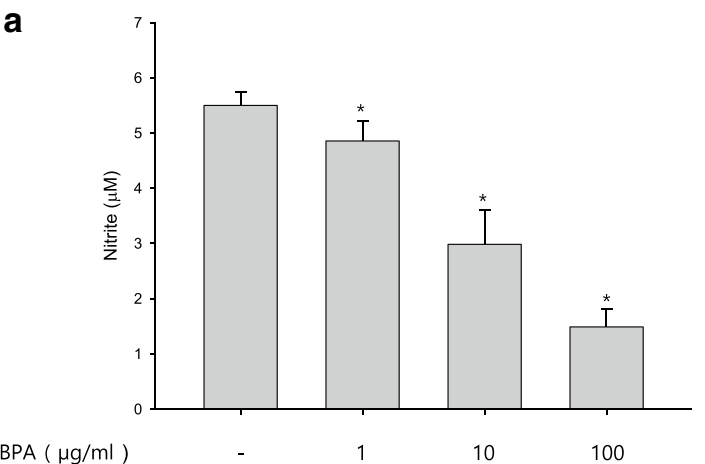

C
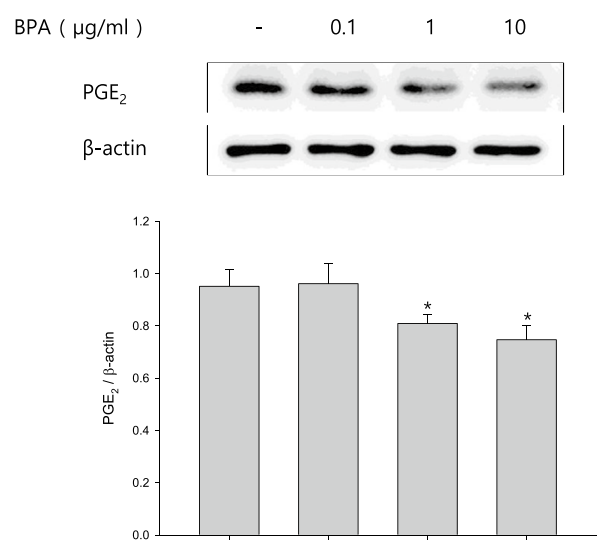

b

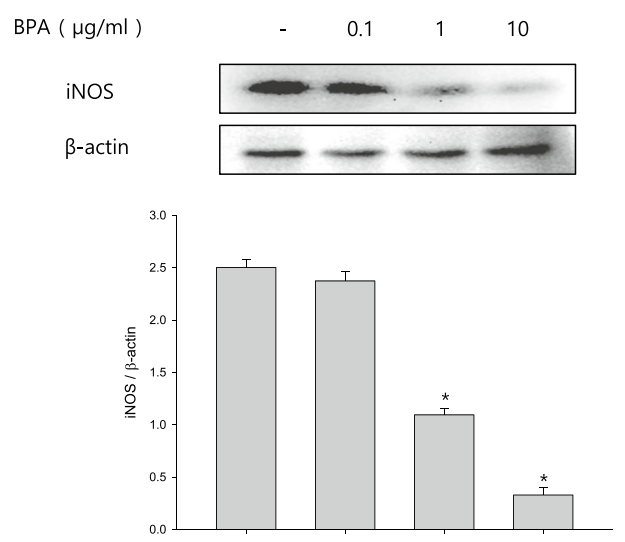

d
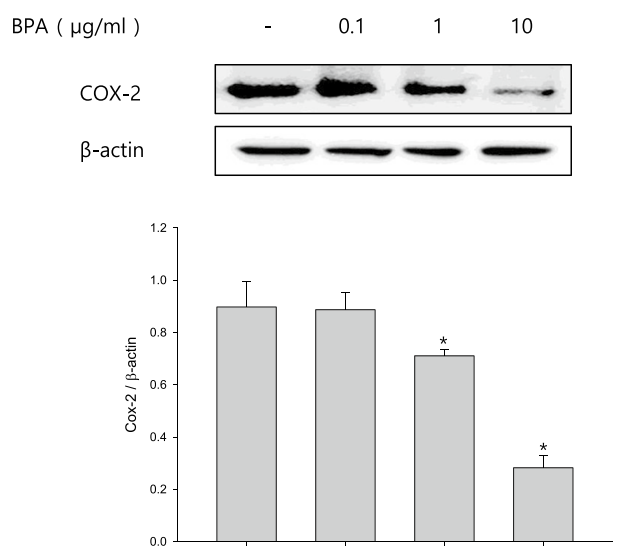

Fig. 2 Effect of BPA on the production of $N O$ and PGE 2 in RAW 264.7 macrophages. Macrophages were treated with different concentrations of BPA $(0.1-10 \mu \mathrm{g} / \mathrm{mL})$ for $24 \mathrm{~h}$. a The amount of nitrite in the medium was investigated using the Griess reaction. Expression levels of $\mathbf{b}$ iNOS, $\mathbf{c}$ PGE2, and d COX-2 were determined by Western blot assay. $\beta$-actin protein levels were used as an internal control. Data are expressed as the mean \pm S.E.M of three independent experiments. ${ }^{*} p<0.05$, significantly different from the untreated group 
a

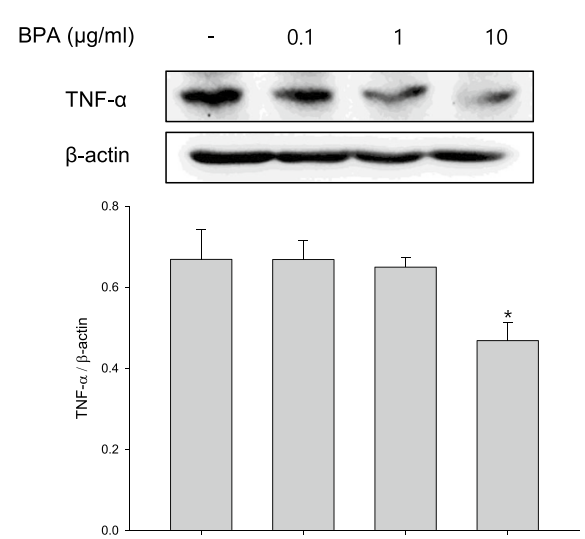

BPA $(\mu \mathrm{g} / \mathrm{ml})$
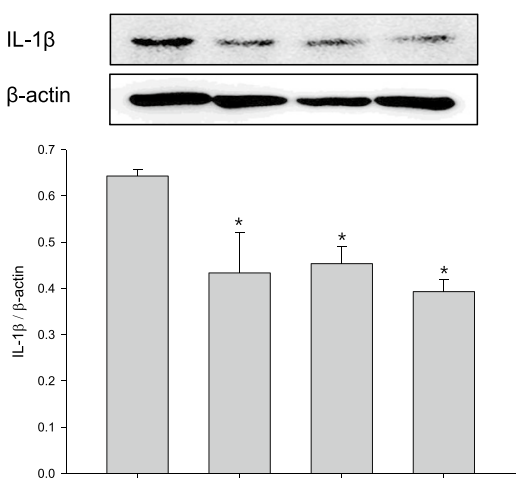

b

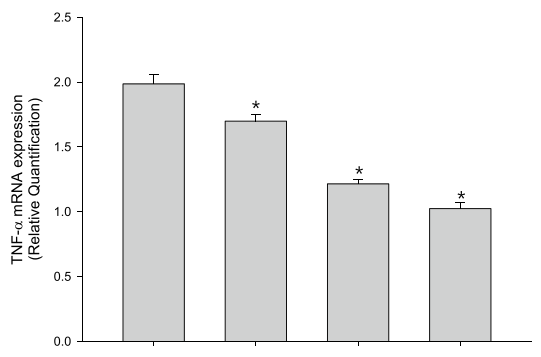

BPA $(\mu \mathrm{g} / \mathrm{ml})$

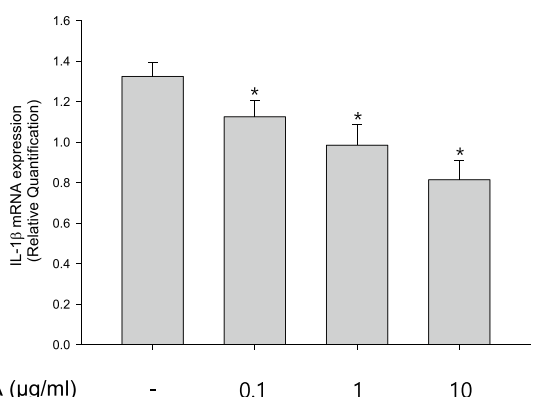

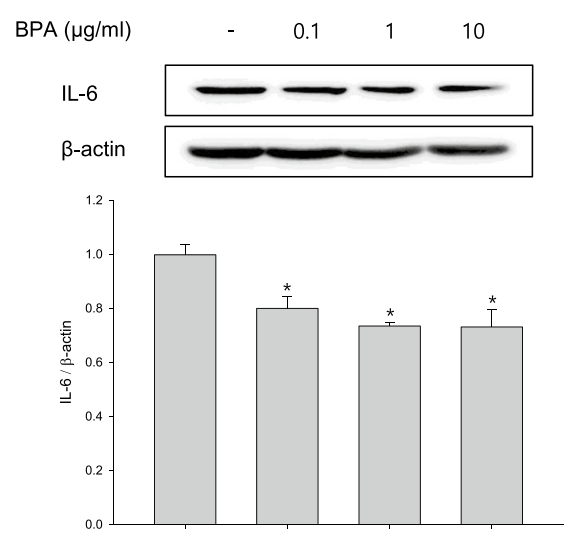

BPA $(\mu \mathrm{g} / \mathrm{ml})$
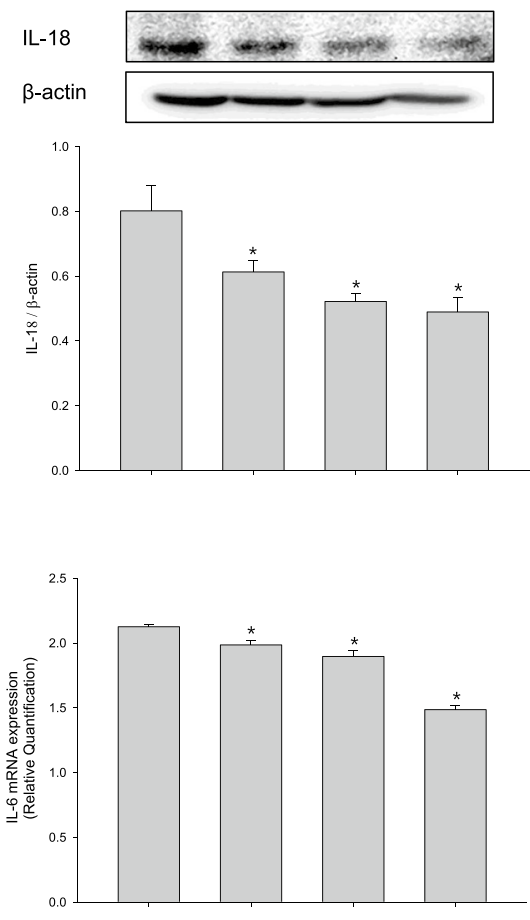

$\mathrm{BPA}(\mu \mathrm{g} / \mathrm{ml}) \quad-\quad 0.1 \quad 1 \quad 10$

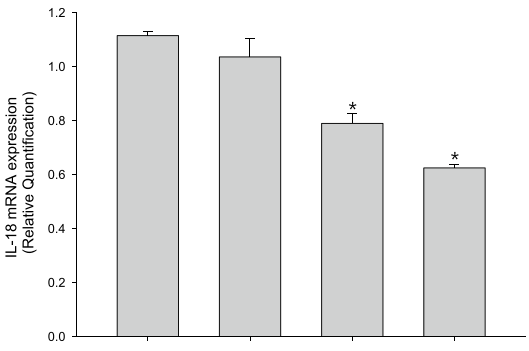

$\begin{array}{lllll}\mathrm{BPA}(\mu \mathrm{g} / \mathrm{ml}) & - & 0.1 & 1 & 10\end{array}$

Fig. 3 Effect of BPA on the protein and mRNA expression levels of TNF- $a, I L-6, I L-1 \beta$, and IL-18 in RAW 264.7 macrophages. RAW 264.7 macrophages were treated with the indicated concentrations of BPA $(0.1-10 \mu \mathrm{g} / \mathrm{mL})$ for $24 \mathrm{~h}$. a The protein levels of TNF- $\alpha$, IL- 6 , IL-1 $\beta$, and IL-18 were examined by immunoblot assay. $\mathbf{b}$ The mRNA expression levels of TNF- $a$, IL-6, IL-1 $\beta$, and IL-18 were investigated by RT-PCR. Data are expressed as the mean \pm S.E.M of three independent experiments. ${ }^{*} p<0.05$, significantly different from the untreated group 
shown in Fig. 3b, treatment of cells with BPA attenuated the mRNA expression levels of TNF- $\alpha$, IL- 6 , IL- $1 \beta$, and IL-18 compared to control cells (no BPA treatment).

\section{Effect of BPA on the activation of NLRP3 inflammasome in RAW 264.7 macrophages}

NLRP3 inflammasome is activated by the micro-organisms and endogenous indicators of cellular stimuli, including ATP and leads to the generation of cytokines maturation. To determine the effect of BPA on NLRP3 inflammasome activity, we stimulated macrophages with BPA and ATP to prime NLRP3 activation. We then examined the protein expression levels of NLRP3, ASC, caspase-1, IL-1 $\beta$, and IL-18 using a Western blot analysis (Figs. 4a-d). Treatment with BPA attenuated the expression level of NLRP3 in a concentration-dependent manner. Moreover, ASC expression levels were decreased following treatment with BPA and ATP. BPA treatment also attenuated the release of caspase- 1 and the production of IL-1 $\beta$ in BPA/ATP-incubated macrophages. In addition, we determined the effect of siNLRP3 in BPAincubated RAW 264.7 macrophages (Fig. 4e). The level of secretion cytokines was increased by knockdown of the NLRP3 gene. These data demonstrate that BPA can disrupt IL-1 $\beta$ and IL-18 release by down-regulating NLRP3 activation and caspase- 1 expression.

\section{Effect of BPA on NF-KB activation and MAPK phosphorylation in RAW 264.7 macrophages}

We next examined the effect of BPA on the stimulation of NF-kB activity in RAW 264.7 macrophages. A western blot analysis indicated that BPA suppressed NF- $\kappa B$ p65 translocation from cytosol to the nucleus (Fig. 5a). We also examined whether BPA affected the degradation of IкB $\alpha$. As expected, incubation with BPA induced a significant concentration-dependent inhibition of IкB $\alpha$ degradation (Fig. 5b). However, the decreased levels of cytokines were markedly increased by pre-incubation with an NF- $\mathrm{KB}$ inhibitor followed by BPA treatment. Taken together, these results demonstrate that BPA can decrease the activation of NF- $\mathrm{KB}$, leading to the inhibition of the production of inflammatory mediators (Fig. 5d).

MAPKs activated by lipopolysaccharide are known to act an essential role in the secretion of inflammatory mediation factors and the translocation of p65. Therefore, we determined the effect of BPA on the activation of MAPKs. Macrophages were activated with $0.1-10 \mu \mathrm{g} / \mathrm{mL}$ BPA for $20 \mathrm{~min}$ and activation of the different MAPKs assessed by determining their phosphorylation levels by western blotting. Treatment with BPA attenuated the phosphorylation of both p38 and ERK in a concentration-dependent manner. However, activation of JNK was not affected by BPA (Fig. 5c). To determine whether MAPK signaling pathways are associated with NLRP3 activity and pro-inflammatory cytokine expression in LPS-incubated macrophages, we used the p38 MAPK inhibitor SB203580, the ERK1/2 inhibitor PD98059, and the JNK inhibitor SP600125 to determine their effects on the BPA-induced regulation of NLRP3 inflammasome and pro-inflammatory cytokine level. As shown in Fig. 5e, pre-incubation of RAW 264.7 macrophages with these inhibitors for $2 \mathrm{~h}$ before BPA exposure increased the expression levels of both NLRP3 and pro-inflammatory cytokines. MAPK phosphorylation was remarkably suppressed by BPA in RAW 264.7 macrophages. These finding indicate that activation of MAPKs is related to the production of inflammatory cytokines.

\section{Discussion}

Bisphenol A is well known that it affects neurological diseases as well as cancer, obesity and diabetes. However, the immunosuppressive effects of BPA on immune responses in various diseases are not well known. Therefore, this is the first report finding the immunosuppressive effects of BPA that inhibits the activation of inflammation factors by suppression MAPK and NF-kB pathway and NLRP3 inflammasome regulation the immune response by inhibiting MAPK and NLRP3.

Inflammation is an immune response involving various signaling pathways. The inflammatory response is a necessary and beneficial mechanism for responding to diseases. It can however also cause several diseases including asthma, arteriosclerosis, and cancer $[12,13]$. Therefore, it is very important to control the immune response appropriately. Inflammatory responses in macrophages have an important function in the overall immune response [14]. When macrophages are confronted with stimuli such as PAMPs and DAMPs, they initiate an immune response by producing pro-inflammatory cytokines, as well as inflammatory mediators [15-17]. These pro-inflammatory

\footnotetext{
(See figure on next page.)

Fig. 4 Effect of BPA on ATP-primed NLRP3 inflammasome activation and the expression of IL-1 3 and IL-18 in RAW 264.7 macrophages. a Macrophages were treated with various concentrations of BPA $(0.1-10 \mu \mathrm{g} / \mathrm{mL})$ for $7 \mathrm{~h}$ and then treated with ATP for $1 \mathrm{~h}$. $\mathbf{b}-\mathbf{d}$ RAW 264.7 macrophages were treated with several concentrations of BPA for $23 \mathrm{~h}$ and incubated with ATP for $1 \mathrm{~h}$. Whole cell lysates were investigated by western blot assay. e RAW 264.7 macrophages were transfected with siNLRP3 and treated with BPA for 23 h and then incubated with ATP for 1 h. Data are expressed as the mean \pm S.E.M of three independent experiments. ${ }^{*} p<0.05$, significantly different from the untreated group
} 
a

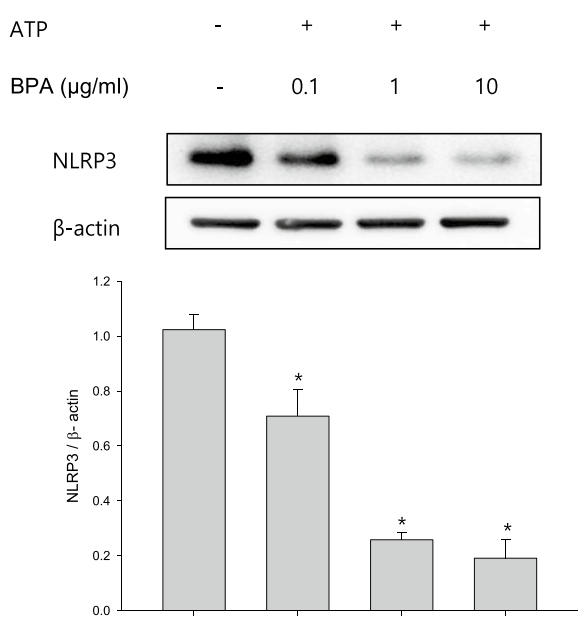

C

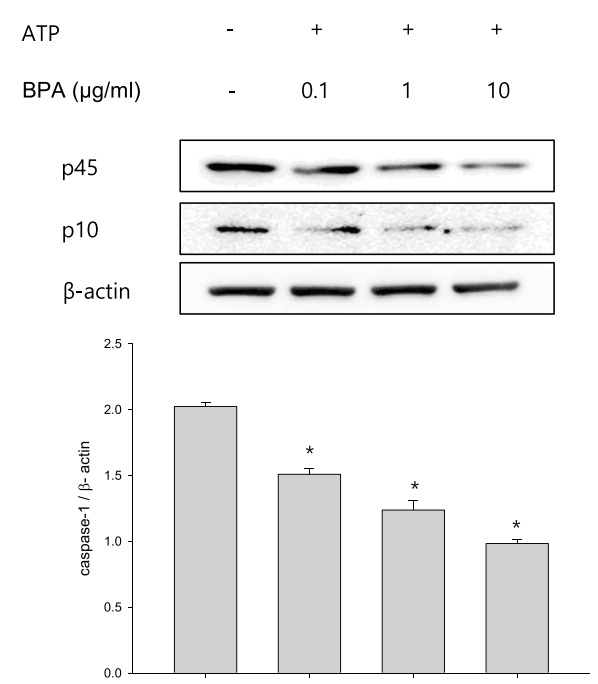

b

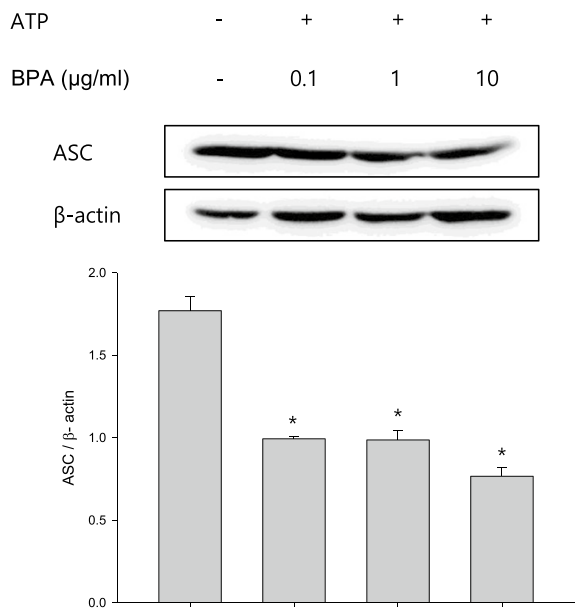

d

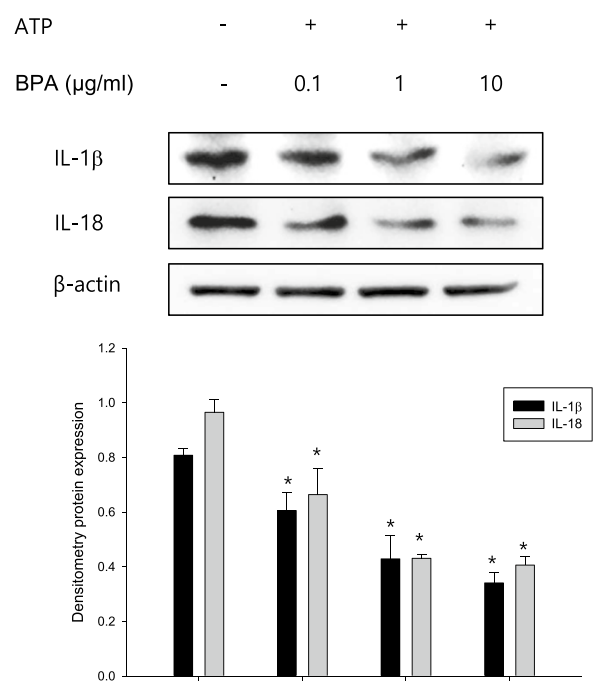

e

SiNLRP3 $(50 \mathrm{nM})$

BPA $(10 \mu \mathrm{g} / \mathrm{ml})$

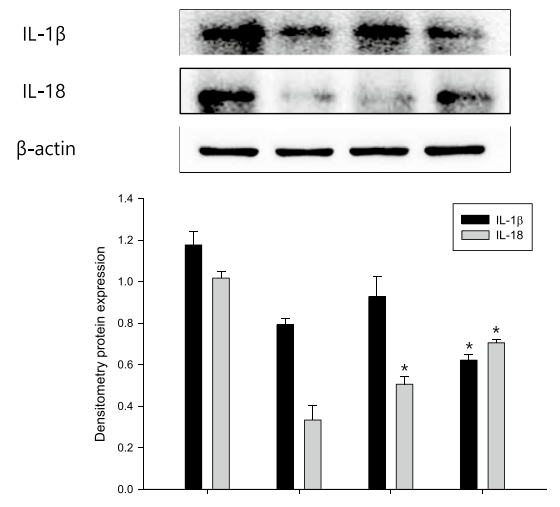


(See figure on next page.)

Fig. 5 Effect of BPA on NF-KB activation and MAPK phosphorylation in RAW 264.7 macrophages. a Cells were activated with BPA $(0.1-10 \mu \mathrm{g} / \mathrm{mL})$ for $4 \mathrm{~h}$. The phosphorylation levels of p 65 were detected by western blot assay to analyze the translocation of NF-kB. $\mathbf{b}$ Macrophages were incubated with various concentrations of BPA for $4 \mathrm{~h}$. Whole cell lysates were investigated by western blot assay using an anti-IkBa antibody. c RAW 264.7 macrophages were treated with indicated concentrations of BPA $(0.1-10 \mu \mathrm{g} / \mathrm{mL})$ for $20 \mathrm{~min}$. The phosphorylation levels of MAPK were detected by western blot assay. d RAW 264.7 macrophages were incubated with the NF-KB inhibitor Bay 11-7082 for $2 \mathrm{~h}$ and then stimulated with BPA for $4 \mathrm{~h}$. e RAW 264.7 macrophages were treated with BPA for $20 \mathrm{~min}$ in the absence or presence of the p38 MAPK inhibitor SB203580 (10 $\mu$ M), the ERK1/2

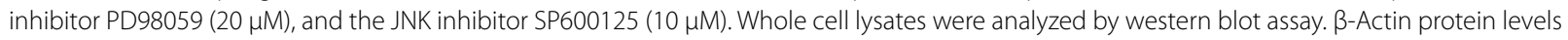
were used as an internal control. Data are expressed as the mean \pm S.E.M of three independent experiments. ${ }^{*} p<0.05$, significantly different from the untreated group

cytokines have a critical role in the host innate and adaptive immune responses. MAPKs and NF-kB are known to have essential roles in the production of $\mathrm{NO}$ and $\mathrm{PGE}_{2}$ as well as in the release of pro-inflammatory cytokines such as TNF- $\alpha$, IL-6, IL- $1 \beta$, and IL-18 [18, 19]. For these reasons, we examined effect of BPA on the mediation of inflammatory factors and cytokines in macrophages. We also assessed the effect of BPA on the activation of the NLRP3 inflammasome in the inflammatory response.

Our data suggest that BPA significantly decreases the production of $\mathrm{NO}$ and $\mathrm{PGE}_{2}$ in RAW 264.7 macrophages by inhibiting the expression of iNOS and COX-2. These data suggest that BPA can block inflammatory response mediated by inflammatory mediators including $\mathrm{NO}$ and $\mathrm{PGE}_{2}$. In addition, the production of inflammatory cytokines was decreased by BPA treatment. MAPK signaling pathway is known to be essential for many metabolic processes such as cell differentiation and cell division [20,21]. It is also very important in mediating inflammatory responses. The NF- $\mathrm{kB}$ signaling pathway is downstream of the MAPK signaling pathway and is a central mediator of pro-inflammatory gene induction and responses [22]. We found that phosphorylation of MAPK is inhibited by BPA, leading to a decreased degradation of IkB $\alpha$ and the subsequent down-regulation of p65 activation in RAW 264.7 macrophages. These data confirm that MAPK acts upstream of NF-KB in the inflammatory response in macrophages.

The formation of the inflammasome complex is triggered through pathogen-related molecular patterns (PAMPs) or danger-associated molecular patterns (DAMPs) recognized by the cell. Inflammasomes are well-assembled platforms for inflammation signaling containing platform, adaptor, and effector protein. When macrophages are caused inflammatory reaction by a stimulation, specific receptor recruits ASC containing the caspase activation and recruitment domain (CARD) and pro-caspase 1. At this time, the pyrin domains of the platform protein and the adaptor protein form a bond, and then the CARD of the effector protein is bound to them $[6,11]$. The mature of pro-caspase 1 results in the production and proteolytic activity of IL- $1 \beta$ and IL-18. Specially, IL- $1 \beta$ is known as a gatekeeper cytokine that acts more specifically on the activity and mediation of inflammation [23].

The NLRP3 inflammasome can be stimulated through various stimuli as part of the inflammatory response in immune cells. For example, in macrophages, the NLRP3 inflammasome is primed by several stimuli including microorganisms, crystal, and ROS. It is well known that ATP is required for the activity of the NLRP3 inflammasome $[7,8]$. Our data show that treatment with BPA downregulates the activation of the NLRP3 inflammasome. Furthermore, BPA decreases the activation of caspase-1 in stimulated Macrophages. As a result of the suppressed expression of NLRP3 and the maturation of caspase-1, IL- $1 \beta$ and IL-18 are not released. Therefore, we confirmed that the phosphorylation of caspase- 1 through activation of the NLRP3 inflammasome regulates cytokine release, especially of IL-1 $\beta$ and IL-18. In general, it is important to find the optimal balance between inflammatory activation and inflammation inhibition for the appropriate moment and an adequate degree of inflammatory response [10, 24]. This depends on the overall inflammation regulation mechanism, and inflammasomes also regulate the activity of the inflammatory mechanism through structural and functional differences.

In summary, these finding suggest that BPA has a profound effect in disturbing the immune responses of macrophages. It can attenuate the release of pro-inflammatory cytokines and inflammatory mediators by the MAPK and NF- $\mathrm{KB}$ signaling pathways in macrophages. In addition, BPA changes the effect on the inflammatory response by regulating the activation of inflammasomes. Thus, our finding demonstrate that BPA plays a critical disturbing role in immune responses of macrophages. 
a

BPA $(\mu \mathrm{g} / \mathrm{ml})$
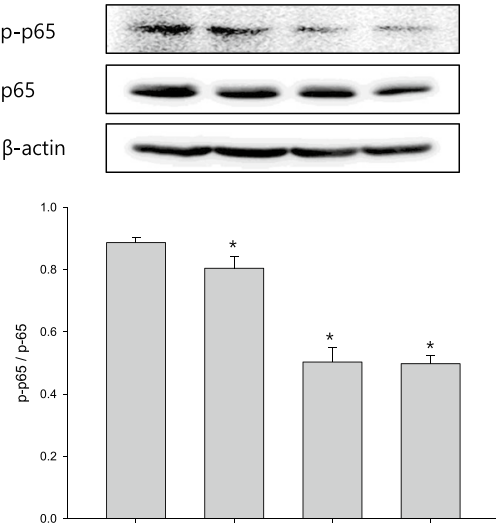

C

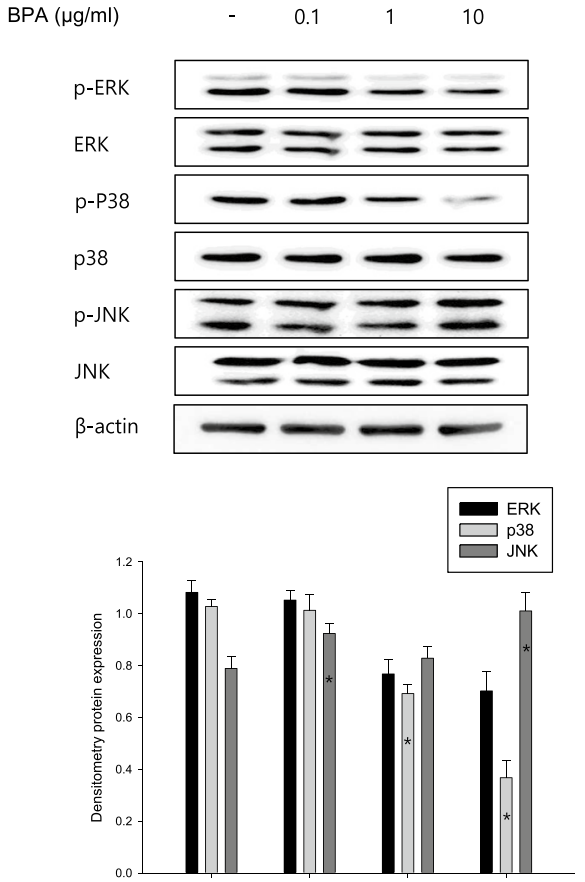

b

$\mathrm{BPA}(\mu \mathrm{g} / \mathrm{ml}) \quad-\quad 0.1 \quad 1 \quad 10$
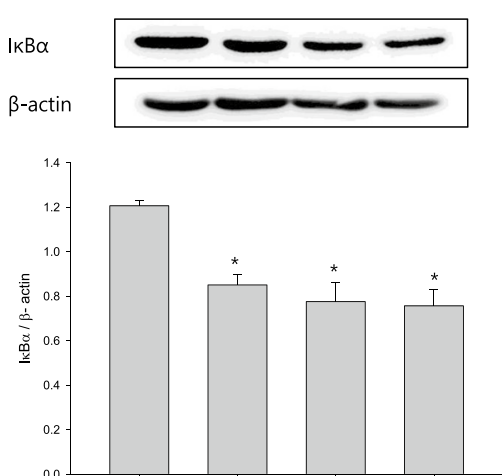

d

Bay $11-7082(10 \mu \mathrm{M})$

BPA $(10 \mu \mathrm{g} / \mathrm{ml})$

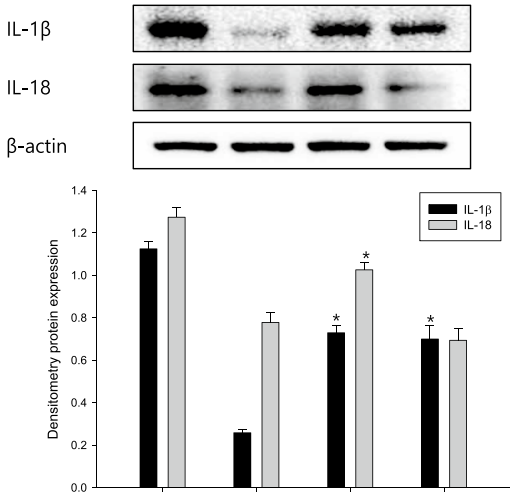

e

PD98059

SB203580

SP600125

BPA $(10 \mu \mathrm{g} / \mathrm{ml})$
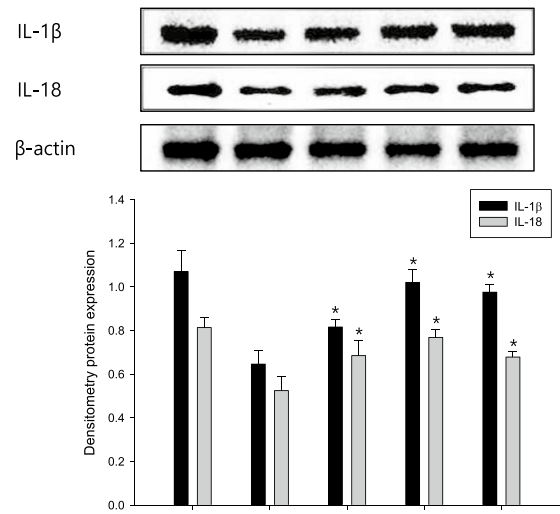


\section{Abbreviations}

BPA: Bisphenol A; EDCs: Endocrine-disrupting chemicals; NLRP3: Nod-like receptor protein 3; ASC: Apoptosis-associated Speck-like protein containing a Caspase-recruitment domain; Caspase-1: Cysteine-aspartic proteases-1; $\mathrm{PGE}_{2}$ : Prostaglandin $\mathrm{E}_{2}$; Cox-2: Cyclooxygenase-2; NF-kB: Nuclear factor-kappa B; IkBa: Nuclear factor of kappa light polypeptide gene enhancer in B-cells inhibitor alpha; TNF-a: Tumor necrosis factor alpha; IL-6: Interleukin-1 $\beta$.

\section{Acknowledgements}

This research was supported by the Main Research Program (E0164400-04) of the Korea Food Research Institute (KFRI) funded by the Ministry of Science.

\section{Authors' contributions}

HW, SK, and YS conceived and designed the experiments. HW performed the experiments. HW, SK, and YS analyzed data and HW wrote the manuscript. HW, SK, and YS contributed reagents/materials/analysis tools. All authors read and approved the final manuscript.

\section{Funding}

This research was supported by the Main Research Program (E0164400-04) of the Korea Food Research Institute (KFRI) funded by the Ministry of Science.

\section{Availability of data and materials}

All data generated or analysed during this study are included in this published article.

\section{Competing interests}

The authors declare that they have no competing interests.

\section{Author details}

${ }^{1}$ Korea Food Research Institute, 245, Nongsaengmyeong-ro, Iseo-myeon, Wanju-gun, Jeollabuk-do 55365, Republic of Korea. ${ }^{2}$ School of Pharmacy, Sungkyunkwan University, Suwon 16419, Republic of Korea. ${ }^{3}$ Divison of Food Biotechnology, University of Science and Technology, Daejeon, Korea.

Received: 19 August 2020 Accepted: 7 November 2020

Published online: 20 November 2020

\section{References}

1. De Coster S, van Larebeke N (2012) Endocrine-disrupting chemicals: associated disorders and mechanisms of action. J Environ Public Health 2012:713696

2. Casals-Casas C, Desvergne BJArop (2011) Endocrine disruptors: from endocrine to metabolic disruption. Annu Rev Physiol 73:135-162

3. Birnbaum LS, Fenton SEJEhp (2003) Cancer and developmental exposure to endocrine disruptors. Environ Health Perspect 111(4):389-394

4. Safe S (2000) Bisphenol A and related endocrine disruptors. Toxicol Sci 56(2):251-252

5. Rubin BS (2011) Bisphenol A: an endocrine disruptor with widespread exposure and multiple effects. J Steroid Biochem Mol Biol 127(1-2):27-34
6. Strowig T, Henao-Mejia J, Elinav E, Flavell R (2012) Inflammasomes in health and disease. Nature 481(7381):278-286

7. Ogura Y, Sutterwala FS, Flavell RA (2006) The inflammasome: first line of the immune response to cell stress. Cell 126(4):659-662

8. Caffrey DR, Fitzgerald KA (2012) Immunology. Select inflammasome assembly. Science 336(6080):420-421

9. Schroder K, Zhou R, Tschopp J (2010) The NLRP3 inflammasome: a sensor for metabolic danger? Science 327(5963):296-300

10. Lee H-W, Lee CG, Rhee D-K, Um SH, Pyo S (2017) Sinigrin inhibits production of inflammatory mediators by suppressing NF-KB/MAPK pathways or NLRP3 inflammasome activation in macrophages. Int Immunopharmacol 45:163-173

11. Schroder K, Tschopp J (2010) The inflammasomes. Cell 140(6):821-832

12. Xu J, Huang G, Guo TL (2016) Developmental Bisphenol A exposure modulates immune-related diseases. Toxics 4(4):23

13. Thompson PA, Khatami M, Baglole CJ, Sun J, Harris SA, Moon EY et al (2015) Environmental immune disruptors, inflammation and cancer risk. Carcinogenesis 36(Suppl 1):S232-S253

14. Chawla A, Nguyen KD, Goh YPS (2011) Macrophage-mediated inflammation in metabolic disease. Nat Rev Immunol 11:738

15. Arango Duque G, Descoteaux A (2014) Macrophage cytokines: involvement in immunity and infectious diseases. Front Immunol 5:491

16. Liu Y, Mei C, Liu H, Wang H, Zeng G, Lin J et al (2014) Modulation of cytokine expression in human macrophages by endocrine-disrupting chemical Bisphenol-A. Biochem Biophys Res Commun 451(4):592-598

17. Mills C (2012) M1 and M2 macrophages: oracles of health and disease. Crit Rev Immunol 32(6):463-488

18. Song H, Park J, Bui PTC, Choi K, Gye MC, Hong YC et al (2017) Bisphenol A induces COX-2 through the mitogen-activated protein kinase pathway and is associated with levels of inflammation-related markers in elderly populations. Environ Res 158:490-498

19. Lee YJ, Han JY, Lee CG, Heo K, Park SI, Park YS et al (2014) Korean Red Ginseng saponin fraction modulates radiation effects on lipopolysaccharide-stimulated nitric oxide production in RAW264.7 macrophage cells. J Ginseng Res 38(3):208-214

20. Rao KM (2001) MAP kinase activation in macrophages. J Leukoc Biol 69(1):3-10

21. Herlaar E, Brown Z (1999) p38 MAPK signalling cascades in inflammatory disease. Mol Med Today 5(10):439-447

22. Tak PP, Firestein GS (2001) NF-kB: a key role in inflammatory diseases. J Clin Investig 107(1):7-11

23. Sutterwala FS, Haasken S, Cassel SL (2014) Mechanism of NLRP3 inflammasome activation. Ann NY Acad Sci 1319:82-95

24. He Y, Hara H, Núñez G (2016) Mechanism and regulation of NLRP3 inflammasome activation. Trends Biochem Sci 41(12):1012-1021

\section{Publisher's Note}

Springer Nature remains neutral with regard to jurisdictional claims in published maps and institutional affiliations.

\section{Submit your manuscript to a SpringerOpen ${ }^{\circ}$ journal and benefit from:}

- Convenient online submission

- Rigorous peer review

- Open access: articles freely available online

- High visibility within the field

- Retaining the copyright to your article

Submit your next manuscript at springeropen.com 